\title{
A Helpful Analysis of the Technological Mix based on HPC and Artificial Intelligence for Maintaining Competitiveness in the Business Environment
}

\author{
Vasile MAZILESCU ${ }^{\star}$
}

\begin{tabular}{l}
\multicolumn{1}{c}{ A R T I C L E I N F O } \\
\hline Article history: \\
Accepted March 2020 \\
Available online May 2020 \\
JEL Classification \\
M15, C80 \\
Keywords: \\
HPC, AI, Cloud Computing, TM, 5G
\end{tabular}

\section{Introduction}

Business processes play a vital role in the smooth running of an enterprise as well as in its evolution. A well planned and strategically designed business process encourages the business by reducing human expenses, risks and errors, improving efficiency, decisively targeting the customer (continually informing the company about customer wishes, products, service comments), filling communication gaps, more rigorous time management, the adoption of new technologies (the business process continues to change and improve over time). Two major factors determine the HPC extensions, including business. One is the increasing of data volumes. Another extremely important aspect that drives the extension of the HPC scope is the increasing computing power at affordable costs and the ability to extract knowledge from growing data volumes to support new use cases. This has spurred fierce research into new AI algorithms. In addition to the traditional analysis required to gain knowledge about simulations, HPC are fueling a new generation of convergent technological mixes that integrate AI by creating models and simulation tasks closer to the expected utility $[1,2]$. HPC platforms ensure increased performance and efficiency of calculations for training deep learning models by processing real-time data flows specific to different business processes and creating AI-based solutions that require large amounts of resources $[3,5,34]$. HPC refers to systems that, through a combination of processing capacity and storage capacity, can quickly solve difficult computing problems in a wide range of scientific, engineering, medicine and business fields. HPC is a strategic technology, with extraordinary economic competitiveness and with implications for different security forms. HPC simulation allows product developers to improve the design and simulate the performance of many critical components before a physical prototype is tested in an expensive simulation environment. Designers can use computational modeling and simulation to explore various design options before constructing physical prototypes and performing experimental tests $[4,6]$. 
The current sets of applications are concentrated, for example, in presenting the products online to a consumer, based on their sales history. However, AI can add many dimensions to such recommendations by conducting sentimental analyzes and changing trends to make relevant searches [9]. Consumers' online retailing experience tends to be affected by the large number of product alternatives available, along with different sets of variants in each product segment. The paradox of the choice leads to aggravation of the confusion of the potential consumers who are going to buy their initial sets of retail purchases. In addition, search engines are not well tuned to provide expected search results based on consumer preferences. From this point of view HPC and Cloud Computing technology can be successfully used in the SCM domain [10,12]. These systems help to ensure the transparency and visibility of the processes, because the use of these technologies presents the advantages of scalability, data integration and interoperability. All partners in the global supply chain can be integrated into an online social network with real-time information on all the subsystems. The implementation and use of a TM based on HPC, cloud and AI results in the transition from the connection between the physical processes (inventory, storage or transport) to the processes based on information and knowledge in the operations of the supply chain. ICT progress creates new opportunities when suppliers, business partners and customers collaborate to create value. The use of TM significantly improves the integration of the supply chain process, leading to better business performance [7].

This paper consists of the following sections: Section 2 highlights a literature review based on current characteristics of the smart enterprise, based on continuous digitization. The idea of including TM in business processes is supported in a selective, rational and aggressive way. Section 3 details TM based on HPC and AI, presenting a series of attributes of these technologies, successfully involved in more and more business processes, including SCM. In section 4 the characteristics of the current mobile ecosystem based on the $5 \mathrm{G}$ unified model, which already affects every industry and business area, will be briefly resumed, will make the logistics more digitalized and transparent for all actors involved, change the business logic and the labor relations. The conclusions of the paper are certainly in favor of the creation and development of TMtype infrastructures, which are becoming stronger and more flexible.

\section{Literature Review}

In this era of continuous and bitter digitization, enterprises improve all their operations, from the product design process, the supply chain and to the delivery. Industry in general and retail in particular is one of the largest and most profitable industries in the world. The industrial sector has grown the most over other global sectors and has experienced a revolution in recent years. The activity of the retail industry has also increased steadily with the increase in demand based on population growth. The implementation of AI at the level of many business processes and of any large retailer in particular, enhances the comparative advantage of the company both in terms of keeping the retail market and the base of consumers anywhere [13,22]. Different types of AI applications have been developed in terms of improving services and retail experience for consumers. Retail, marketing and sales represent the area with the most important potential value in $\mathrm{AI}$, the promotion and management of customer services are the main areas targeted $[18,23,26]$. Using customer data to personalize promotions, including tailoring individual offers every day, can lead to significant sales growth. In the field of consumer goods, SCM is the key function that benefits from the implementation of AI. Among the use cases, we see how forecasting based on the underlying causal factors of the demand, rather than the previous results, can improve the accuracy of the forecast, which translates into a potential reduction of inventory costs and revenue growth [5]. Due to the importance of risk assessment and management in the banking sector, for example for loan underwriting and fraud detection, AI has much greater potential to enhance the performance of risk mitigation in the banking sector [4]. From machines equipped with AI-based control systems to program-driven vehicles, improved data analysis and bug detection software, smart technology makes value chains more digitalized than ever before. [10] In order to make the most of AI in the supply chain, adopting natural language processing (NLP), robotic process automation (RPA) and deep learning technologies are essential $[16,17]$. An important capacity is represented by the use of data that are not available in a relational format or that cannot be analyzed with traditional methodologies. The use of this data requires new approaches that can efficiently manage the volume and heterogeneous types of data, which must be presented in a flexible and compatible format specific to AI approaches. Other benefits of the AI approach include predictive analytics that provide key insights to optimize the performance of the supply chain. AI can have positive implications both in terms of increasing the consumer experience and in increasing the efficiency of the sustainable growth of the companies' revenues, by maintaining without error of its different sets of operations [29]. HPC is a practical solution for using servers, clusters and supercomputers, along with software packages, tools and related computing tasks. These computing tasks benefit from GPU chips, as they are tightly coupled with accelerated hardware, resulting in performance improvements a few times. Many of the recent advances in the implementation of AI-based systems can be attributed to the massive increase in computing capabilities, through the development of HPC platforms, reconfigurable, scalable and increasingly powerful [31,32,35]. Regulating these computing resources can become one of the main challenges for AI. The future of developing robust AI solutions requires HPC platforms designed for parallel computing. An HPC infrastructure allows the organization to scale and create ML algorithms that can use huge amounts of data. HPC is a way of aggregating multiple computers and 
storage devices to solve problems of high algorithmic complexity [15]. In an HPC environment, the nodes work together to solve a larger problem than any computer can currently solve. Many industries use HPC technology today for daily business [14,21,24]. Retailers run AI models to analyze customer data and to provide purchase recommendations. HPC brings additional benefits of SCM scalability, integration, portability, processing power, storage and interoperability. Mega corporations and retail giants have implemented HPC solutions for SCM and have achieved efficient data management and analysis. The HPC space changes, and these changes create their share of challenges. HPDA (High Performance Data Analysis) and AI-based methods are analyzed and developed in an extremely fast and practical way. They complement the computing mix of traditional HPC users. These methods also motivate a growing number of commercial firms to integrate HPC systems into enterprise data centers to advance business operations, in particular fraud detection and cyber security, business information, marketing, ERP and sales planning. Firms are determined by the global competitive market to develop this TM based on HPC and AI, which is essentially the immediate need to ask more complex questions to their data structures and to obtain real-time answers [27,28]. Unlike previous approaches, AI is enormously involved in developing the capacity of HPC systems beyond a series of rule-based and cause-effect relationships. Instead, AI analyzes data using a set of instructions, theories and algorithms. Learning from these theories, AI can analyze in a predictive regime and can better understand the context using logical inferences to complete a series of anomalies due to the characteristics of large data sets or knowledge [19,25]. AI models better and more traditionally complement traditional HPC solutions to more quickly and comprehensively reveal a range of dynamic features of the problem to be solved, than data-based and analytical applications, however complex they may seem current time. As capabilities based on HPC (simulation, modeling, big data analysis) and AI converge, hybrid workloads are becoming more common. One step in a hybrid workload might involve modeling and simulation, while the next step requires deep learning ability. The varied nature of these workflows can be a direct challenge for non-optimized HPC systems. However, a balanced multi-node system, excellent for HPC and AI, reveals new possibilities for automated discovery and can work effectively for the organization and people, by discovering new operational knowledge. Improving the relationships between the supplier buyer and access to actionable knowledge in an appropriate manner, as well as managing the dialogue and coordination between different actors are other benefits. This greatly increases the company's ability to respond to market dynamics. The perfect access to information, the visibility and the transparency of the data processed in an intelligent way increases the efficiency of the different processes of the supply chain. Automated identification technologies, such as bar coding and RFID, have made inventory management efficient and have created visibility and transparency of stocks, and transport management systems have optimized routes for shippers.

\section{HPC and AI Convergence, the current Solution for TM}

The company is seen as a system in a permanent search for optimal solutions to achieve its business objectives. The Intelligent Enterprise (IE) model as a system model, supported by the globalization of the market, is a trend that supports the effort to approach the concept of automated factory. IE permanently seeks to harmonize the manufacturing system with the requirements of the external environment. The main objective of a production system is a rapid response, which involves increasing flexibility, ensuring high quality, reducing the manufacturing cycle per product and the production costs. The pressure is given by the market and by the technological changes. Major leaps were made with the evolution of computer support based on HPC and AI and flexible automation and robotization. The information processing subsystem ensures the acquisition of the specific data for the integration of the company in the business environment and its adaptation to the social and technological macro context. So many concepts illustrate, with a multitude of arguments, the processes of valorization of the knowledge in the enterprise, of the intellectual capital (composed of experience and competence), of the knowledge of processes and clients, of renewal and development. It develops a co-production of the processes, with the client's involvement in the elaboration of the goods that he wants, by ensuring the quality or by defining the quality standards. Another way to develop this approach is to involve the external partners of the company. In this case we can talk about a co-piloting of the jointly designed project, that is, a way of managing the project in which the potential interested actors collaborate in designing a common multidimensional representation of it, and to establish the principles of general action. Moreover, competitors can be associated in a collaborative manner. The employee ceases to be seen as a labor force, now being recognized as having experience and skills, so the decision can be decentralized. Optimizing decisions requires combining and coordinating the experience of different software or human agents. The concept of organization appears as a central element in structuring the activities of decentralized human groups as well as in solving problems in multi-agent systems, hence the idea of integrating these fields. Considerations on the evolution of the enterprise (having as object the changes of the economic environment, of the production systems, of the organizational structures) indicate that at this moment the features of a different enterprise are foreshadowed.

External factors with an impact on the company are growing faster and faster, their dependencies are increasingly emphasized where and the need for the company to be able to change their behavior more quickly, to respond in an appropriate manner. On the other hand, competitiveness (involving innovation, anticipation, speed of response, agility) is a gradually constructed concept, a transition from quantitative to qualitative, initially towards 
the outside of the company and then within it. Under these conditions, the design of the company in the future is done through simulation and anticipation, so it is necessary that the company can dynamically modify the objectives for the adequacy of the strategies. Moving to a high complexity requires the development of collaborations and decision autonomy. Strategic actors are becoming more numerous, there is no longer a dominant one and negotiation becomes necessary. Which requires and multiplies the processes of involvement and co-optation, of motivating the whole staff, which is thus placed very frequently in communication situations, to be able to work together in teams, to exchange experience, to learn. It is necessary to use the entire organizational intelligence, requiring the effective exploitation of the internal potential. Starting from the limits presented above, we can define IE starting from its basic objectives and taking into account the major impact and implications of the AI in this approach. In an IE there are several information systems integrated with tools for collecting and analyzing knowledge. These solutions allow the company to improve its customer service, relationships with partners and create products based on knowledge obtained from the internal data of the company. The smart enterprise is an organization that needs to function efficiently, using a series of knowledge-sharing models. ICT support allows people to store their learning and simulation experience and to increase their understanding of the production and processing of certain real events. The smart enterprise consists of complex networks of people, knowledge (internally tacit and externally coded explicitly), infrastructures and information processes that distribute the critical knowledge (needed) to various consumers. All these components together allow the achievement of the objectives of any enterprise, in the form of chains of processes (production, sales, procurement, the promotion of services). An effective IE requires much more than these components: people need a culture that allows them to collaborate, integrated 5G electronic networks require a compatible context and content, the implementation of different components requires a permanent adaptation of a TM based on HPC and AI, to cope with high competitiveness. An important aspect in defining IE is connectivity based on the ability to convert primary data from various sources into accurate and rapid knowledge and decisions. A smart company connects experiences and logical reasoning processes using knowledge exploitation solutions, business platforms and application packages focused on the organization's objectives [11].

Cloud and associated technologies such as virtualization and Software as a Service (SaaS) is the next major leap and game changer for businesses. With more businesses operating their manufacturing and services to low-cost hubs, poor infrastructure and transport networks, and visibility has become a major challenge. Cloud can integrate all partners in this increasingly global supply chain into an online social network, such as the community, with real-time information on all elements of the supply chain [35]. More solutions are now available and many businesses have exchanged good results. Areas of application where cloud-based solutions are available include demand forecasting, demand planning, electronic procurement, distribution, inventory, storage and transportation systems. In order to fulfill the orders, the retailer must manage the level of stocks in the warehouses. Reordering and reloading stocks are automated using web services for spectacular results. Business analysis facilitates the creation of new perspectives on business performance, using data, statistical methods, quantitative analysis and predictive modeling. Advanced analysis is used for several processes in the planning and execution of the supply chain, such as demand forecasting, inventory management, production and distribution planning. From initiating product search on the retail site to resolving logistics issues and delivering these products to end users, AI can operate on many parameter settings associated with different platform-level algorithms. Moreover, to optimize consumer transactions and to help the company improve its revenue generation capabilities by increasing customer satisfaction, the role of AI tends to be crucial. By implementing programs based on ML algorithms that evolve based on the available data sets, better sets of predictions can be made regarding changing consumer preferences and online activity. The machine learning programs are not application specific and tend to be modified according to the data made available and analyze the data structure to decide the degree of optimization. AI, through Reinforcement Learning can also contribute to the modernization of the security profile of online sites, by increasing the degree of surveillance of suspicious activities in its network. The initial alignment between the $\mathrm{AI}$ and the online site requires the installation of additional sets of servers that tend to host additional random-access memory reconfiguration sets. The high growth prospects of the AI, together with the applicability in terms of collecting and synthesizing behavioral analytical data results in the exponential growth of the income generating capacity.

Given the estimates of demand and sales, the evaluation of investments can be appreciated through AI applications that will highlight the possible restrictions on such approaches. Moreover, reducing computer network security by deploying ML on system servers will aggravate the detection rate of previously undetected malware in the system. Companies should install HPC platforms and supercomputer hardware infrastructure on their networks, before which they must recalibrate their existing IT infrastructure [30]. There must be in the new architectural conception the component for the analysis of unstructured data (type Apache Unstructered Information Management), the component for processing Big Data sets in a distributed computing environment, operating system with multi-core processors with tens or hundreds processing capacities terabytes, natural language processing components, etc. The bandwidth extension should be initiated for peripherals, coupled with the extension of cloud-based services to ensure base growth. Only cost 
reduction at existing installations cannot be the only source of installation cost coverage. There should be a source of cash flow to further capitalize on R\&D and AI expenses that will be borne by the company. Behind the shopping sites, AI is redefining the shopping experience for millions of online shoppers and merchants. For users, some of these enhancements may seem subtle: product search results that have become unchangeably accurate; previous search engines that focus on consumer goods and services may not have realized they wanted to; virtual showcases that display information tailored to individual buyers based on their unique features and preferences; chat services for customers who can solve consumer complaints without human intervention (orders that are delivered in hours instead of days). These improvements regarding the product discovery until the decision to buy the product delivery to the after-sales service have significant implications in the daily activity of the people. By freeing up time for teams, AI will allow them to focus on developing differentiated forecasting strategies and responding better to customer expectations while reducing waste. Smarter supply chain management will reduce stock disruption and prevent overcrowding in stores and warehouses. AI creates new jobs for employees, such as data scientists for data processing and demand planners for business expertise. The retail industry is undergoing a transformation driven by customers expecting a perfect online-store experience. Customers are looking for tools that can make recommendations or better help them find what they need, they want the whole experience to be faster and more personalized than ever. As a result, many retailers are using cloud technologies to help meet these needs. The positioning of an HPC cloud platform differs significantly from other similar commercial platforms because: 1) it can be an open platform that allows it to be modified and extended to all levels of abstraction, from the cloud service stock to experience new features and services. 2) they can support different types of applied research projects and technology transfer in consortia of universities and companies. 3) they opens the way for the implementation of services and data sets, where companies in conjunction with other research and development entities can use, publish and sell innovative cloud services developed in research projects that involve local and global data sources. 4) they clearly become a real and indispensable facilitator of the processes of collaboration in the sector of production, research, but also among the SMEs that need cooperation projects using common technological platforms and cloud storage solutions. For example, designing a POLARDB distributed storage solution with Intel ${ }^{\circledR}$ Optane ${ }^{\mathrm{T} M}$ DC SSDs and Intel 3D NAND SSDs offers excellent storage efficiency, high QoS, high IOPS, high throughput and consistent performance. Intel® SSDs also help adjust costs and performance. Intel ${ }^{\circ}$ Optane ${ }^{\mathrm{TM}}$ DC SSDs help Alibaba Cloud eliminate data center storage bottlenecks, host larger, more accessible datasets, reduce transaction costs for latencysensitive workloads, and improve the total cost of ownership (TCO) of the POLARDB data center [35].

Modern DL models have been exploited in various fields, including Artificial Vision (AV), NLP, search and recommendation. Functional AI clusters, the workloads that drive these models use software frameworks such as TensorFlow, Caffe, PyTorchand CNTK. A critical issue for the efficient operation of AI applications in the cloud is the characterization of the calculation and data transfer processes of these work tasks. The computing part, which involves both GPU computing and access to memory, is not the biggest bottleneck based on the collective behavior of work tasks. Much of the tasks / workload can be potentially reduced when ported to AllReduce Architecture Exploating NVLink high speed for GPU interconnection, and the average speed of $1.7 \mathrm{X}$ can be achieved when the Ethernet bandwidth is upgraded from 25 Gbps to 100 Gbps [34]. New sales solutions are being created using AI-based data analysis to solve the specific business challenges that retailers face in all parts of the value chain. Accustomed to instant access to e-commerce, customers are looking for opportunities to bypass the line and have direct communication channels to request, troubleshoot and of course shopping. Always in constant motion, today's consumers buy where, when and how they want. Retailers are expected to keep up with the mobility of their customers and offer them customized solutions no matter where they shop. Customers change their personal data, such as location, purchase history, to gain relevant experiences. Buyers anticipate that retailers will provide them the services and products that are needed right now and change their behavior depending on the reason and how they make their purchases.

\section{5G-based Mobile Ecosystem Development supports TM Implementation}

There is a lot of talk about $5 \mathrm{G}$ as consumer technology, but less about how it is set up to benefit businesses. First and foremost, it will certainly improve the experience of remote working and collaboration. Network-related tasks, such as online sales or large file sharing in the cloud, will become faster and more efficient, even on mobile devices, without the need for WiFi, giving businesses huge productivity gains and boosting staff morale. In addition to connecting people, 5G will connect things, greatly expanding the capabilities and application of IoT both in the business field but especially in the industrial field (IIoT), which requires better and faster connectivity. It is estimated that $5 \mathrm{G}$ will allow device-to-device communications for up to one million devices per square kilometer. This opens up huge opportunities for IoT innovation and commercial use, offering businesses a competitive edge in creating and using such technologies. However, if existing businesses do not speed up in preparation for $5 \mathrm{G}$, the resulting gaps will inevitably attract new entrants, generating disruptions that negatively affect mature industries. This is why a sustained approach to planning and investing in 5G technology is needed today. Moreover, many of the new 5G applications will be powered by interconnected ecosystems that cross the traditional supply chain and industry boundaries. 
$5 \mathrm{G}$ networks are developed to meet the demands of an extremely mobile and interconnected economic environment. The existence of more and more human-centered applications and the type of machine will define extremely diverse functional requirements that $5 G$ networks will have to satisfy. Within the 5G architecture, the concept of service-based end-to-end (E2E) network, software-defined network (SDN) and network virtualization (NFV) functions are considered as fundamental pillars for sustaining heterogeneous key performance. 5G offers mobile network operators unique opportunities to provide new services to consumers and all businesses. The $5 \mathrm{G}$ ecosystem should facilitate all manufacturers, solution integrators, network and service providers and small and medium-sized enterprises to compete and cooperate effectively, for example through virtualization, standardized interfaces and protocols or open APIs. Solution manufacturers and integrators need to make available virtualization-based implementations and standardized interfaces: Service Customer(SC): uses services that are offered by a Service Provider (SP operates services using aggregated network services): Communication Service Provider offering traditional telecom services, Digital Service Provider offering digital services such as enhanced mobile broadband and IoT to various vertical industries, or Network Slice as a Service (NSaaS) Provider offering a network slice along with the services that it may support and configure. - Network Operator(NOP): in charge of orchestrating multiple virtualised resources. - Data Centre Service Provider (DCSP): Provides data centre services and designs, builds and operates its data centres [33].

$5 \mathrm{G}$ will offer speed, low latency and connectivity to enable a new generation of applications, services and business opportunities that have never been seen before. There are three broad categories of $5 \mathrm{G}$ use cases [37]: Massive communications to machines - also called the Internet of Things (IoT) which involves connecting billions of devices without human intervention to a scale that has not been seen before. It has the potential to revolutionize modern industrial processes and applications, including agriculture, production and business communications. Highly reliable low latency communications - mission-critical, including realtime device control, industrial robotics, vehicle-to-vehicle communication and safety systems, autonomous driving, and safer transportation networks. Low latency communication also opens up a new world where healthcare, procedures and distance treatment are possible. Improved mobile broadband - offering significantly faster data speeds and a greater ability to maintain the connected world. The new applications will include fixed wireless Internet access for homes, outdoor broadcast applications, without the need for more utilities and greater connectivity for people on the move. For communities, 5G will allow billions of devices to connect to smart cities and smart homes, safer smart vehicles, improve education and health care. For companies and industry, 5G and IoT will never provide a wealth of data to enable them to obtain information about their operations. Businesses around the world today recognize that HPC infrastructure is vital to supporting AI and analytics applications of tomorrow. The future of businesses depends on how quickly these technologies, such as AI, will be adopted. In order to transform businesses, AI solutions must work. Future wireless networks will become intelligent communication entities based on distributed computer architectures. This will enable it to meet the requirements of ultra-reliability, high capacity, extremely low latency and low power consumption, presented by scenarios of emerging applications, such as the industrial Internet of Things in future manufacturing processes [31].

The current tendency to meet some of these requirements is through cell densification, mass transmission with multiple multiple outputs (mMIMO), and higher bandwidth exploitation. The introduction of programmable meta-surfaces to make, for example, smart electromagnetic reflectors, large configurable antennas, using thin meta-materials, has opened very attractive perspectives. These intelligent surfaces can be incorporated into the object of daily life and can be used as distributed platforms for the production of sensors, storage and analog computing with low energy and reduced complexity. Media covered with smart surfaces are the recently proposed radio environments. In smart radio environments, the design paradigm has changed from wireless devices / networks that adapt to the environment (eg propagation conditions), to unique device and environment optimization using reconfigurable smart surfaces (RIS) for the purpose. to move to the final limits of wireless communications. With the rapid development of smart terminals and infrastructures, as well as diversified applications (for example, virtual and augmented reality, distance surgery and holographic projection) with colorful requirements, current 4G networks and future 5G networks will not be able to meet rapidly growing demand. traffic. As a result, efforts in both industry and academia have already been focused on researching 6G networks. Recently, Artificial Intelligence (AI) has been used as a new paradigm for designing and optimizing 6G networks with a high level of information. In the paper [20] a smart architecture for 6G networks is proposed for knowledge discovery, intelligent resource management, automatic network regulation and provision of intelligent services, in which the architecture is divided into four layers: intelligent layer, data awareness and analytical layer, smart control and smart software. AI applications and techniques for 6G networks need to be analyzed in order to efficiently optimize network performance, including mobile edge computing with intelligent capabilities, intelligent mobility and manual management and intelligent spectrum management.

Due to the availability of large-scale digital data sets and massive amounts of computing power, deep learning (DL) algorithms can learn data representations by exploiting multiple levels of abstraction. These ML methods have greatly improved the level of knowledge, such as visual object recognition, speech processing, 
natural language comprehension and machine translation. Although these self-organizing systems can be conveniently formalized within statistical mechanics, their internal functioning remains opaque, as their emergent dynamics cannot be solved analytically. HPC requires connecting computers to process data in parallel, allowing those systems to achieve greater computing power. To do this, HPC uses server clusters or "nodes", which work in parallel to perform complex computations. There is no specific number or range of clusters defined to constitute HPC; rather, HPC is simply classified as computing power aggregation in this way to solve a particular problem. A small group of clusters can be as many as four nodes, and a large group can be thousands of nodes. There is an HPC processor in each node. The processor can use multiple cores in a processor or GPU, where GPUs are particularly well suited for simulation, imaging and modeling. It may also have acceleration cards that use GPUs that are more efficient at processing graphics and images. More cores means more energy consumed. Multi-core models were created to divide workloads, requiring cores to communicate with each other. Computing and storage servers have also been linked into groups to communicate. Components and servers must keep pace with each other. Storage or memory components must power and ingest data in rapid succession, while network components must keep up with the transport of server, storage and locations data. Without taking into account these basic design and design requirements, higher levels of performance cannot be achieved for HPC architectures. Recent research in the field of parallel computing is improving performance in grouped systems. Some companies connect large servers in a single cluster, where the total physical size, energy consumption, or heat output of the computing cluster could become a serious problem.

\section{Conclusions}

The use of a TM presented in this paper has become an innovative and interesting paradigm for industry, business, research, medicine [8], etc. These emerging technologies are rapidly developing fields of research that cover extremely powerful exascale computer systems and architectures required for real-time data collection, analysis and semantic interpretation. The combination of AI and HPC has already been used for testing different software modules. For these areas of research, theoretical contributions and research are needed to offer advanced concepts and technologies, reporting experiences and tutorials on emerging trends, analyzing experiences related to implementation, as well as increasingly robust applications. Many of the recent advances in AI can be attributed to the availability of more computing capabilities. Regulating these computing resources can become one of the main challenges for AI. The future of AI requires HPC platforms designed for parallel computing. HPC is a way of aggregating multiple computers and storage devices into one cohesive, archived material to solve big problems in science, engineering and business. In an HPC environment, individual nodes work together to solve a bigger problem than any computer can easily solve. The popularity of this type of infrastructure is growing rapidly: the global HPC market is expected to grow. While HPC is often thought only conceptually, many industries use HPC technology today for their day-to-day business. Retailers run AI models to analyze customer data and provide targeted purchasing recommendations. These workloads benefit from GPU, as they are tightly coupled with accelerated hardware with a performance improvement a few times. A TM must be understood as a conceptual and practical solution for the use of servers, clusters and supercomputers, plus software, tools, components, storage and associated services for scientific, medical, engineering or analytical tasks that are particularly computationally intensive, utilized memory or data management, with increasing use in the business field.

\section{References}

1. Silvinia Caino-Lores, Jesus Carretero, Bogdan Nicolae, Orcun Yildiz, Tom Peterka (2019): Toward High-Performance Computing and Big Data Analytics Convergence: The Case of Spark-DIY, IEEE Access, Vol. 9, https://ieeexplore.ieee.org /stamp/ stamp.jsp?tp $=$ \&arnumber $=8884083$

2. Borut Roncevic, et al. (2019): Go with the Flow: High Performance Computing and Innovation in the Danube Region, Vega Press Ltd., ISBN: 978-1-909736-11-5, http://www.interreg-danube.eu/uploads/media/approved_project_public /0001/33/ e311b83b587d91d3cee39b5d12 a8aaeaccc9171c.pdf

3. (2020): ETP4HPC'S SRA 4, Strategic Research Agenda for High-Performance Computing in Europe, March 2020, https://www. etp4hpc.eu/pujades/files/ETP4HPC_SRA4_2020_web(1).pdf

4. Björn-Sören Gigler, Alberto Casorati and Arnold Verbeek, (2018): Financing the future of supercomputing. How to increase investment in high performance computing in Europe. https://www.eib.org/attachments/pj/financing_the_future_of_supercomputing_en.pdf

5. Chui, M., Manyika, J., Miremadi, M., Henke, N., Chung, R., Nel, P., \& Malhotra, S. (2018): Notes from the AI frontier: Applications and value of deep learning. McKinsey Global Institute discussion paper, April 2018. Retrieved January, 2020 from https:// www.mckinsey.com/featured-insights/artificial-intelligence/notes-from-the-ai-frontier-applications-and-value-of-deep-learning.

6. Stephen J. Ezell, Robert D. Atkinson (2016): The Vital Importance of High-Performance Computing to U.S. Competitiveness, Information Technology \& Innovation Foundation, http://www2.itif.org/2016-high-performance-computing.pdf

7. Davide Strusani and Georges Vivien Houngbonon, (2019): The Role of Artificial Intelligence in Supporting Development in Emerging Markets. https://www.ifc.org/wps/wcm/connect/32e54505-3bfb-4198-b939-e1e8847715f1/EMCompass-Note-69-Role-of-AI-inEMs.pdf?MOD= AJPERES\&CVID $=m N d P i N f$

8. Duft, G., Siekelova, A., Kolencik, J. (2019): Incorporating cognitive artificial intelligence systems and real-time data analytics in clinical care delivery. Am. J. Med. Res. 2019,6, 61-66.

9. Gary Marcus, (2020): The Next Decade in AI: Four Steps Towards Robust Artificial Intelligence, https://arxiv.org /ftp/arxiv/papers/2002 /2002.06177.pdf 
10. Hau L. Lee, Haim Mendelson, Lauren Blake, Sonali Rammohan (2018): Value Chain Innovation:The Promise of AI white Paper, Stanford Value Chain Innovation Initiative. https://www.gsb.stanford.edu/sites/gsb/files/publication-pdf/vcii-white-paper-value-chaininnovation-promise-ai.pdf

11. Heather Riley, Mohan Sridharan (2018): Non-monotonic Logical Reasoning and Deep Learning for Explainable Visual Question Answering, HAI '18, December 15-18, 2018, Southampton, United Kingdom, Association for Computing Machinery. ACM ISBN 978-14503-5953-5/18/12. https://doi.org/10.1145/3284432.3284456, https://www.cs.bham.ac.uk/ sridharm/Papers/hai18_vqa Explain.pdf

12. Jaehun Lee, Taewon Suh, Daniel Roy and Melissa Baucus, (2019): Emerging Technology and Business Model Innovation: The Case of Artificial Intelligence, J. Open Innovation Technology Mark. Complex. 2019,5, 44; doi:10.3390/joitmc50300

13. Kai Jia, Martin Kenney, Juri Mattila, Timo Seppälä (2018): The Application of Artificial Intelligence at Chinese Digital Platform Giants: Baidu, Alibaba and Tencent, ETLA Reports, ETLA Reports No 81. https://pub.etla.fi/ETLA-Raportit-Reports-81.pdf

14. Obschonka, M., Audretsch, D.B. (2019): Artificial intelligence and big data in entrepreneurship: A new era has begun. Small Bus. Econ. 2019, 1-11. https://link.springer.com/article/10.1007/s11187-019-00202-4

15. Working Group (2014): Applied Mathematics Research for Exascale Computing, US Department of Energy, https://pdfs.semanticscholar.org/08c6/485730d302944967cc6c44762deb2c71c080.pdf

16. P.S.H. de Jong, M. van Steenbergen, J.M.E.M. van der Werf, F.J. Bex, (2017): Reasoning on Architecture Design, Technical Report UU-CS2017-019 December 2017, Department of Information and Computing Sciences Utrecht University, Utrecht, The Netherlands www.cs.uu.nl. http://www.cs.uu.nl/research/techreps/repo/CS-2017/2017-019.pdf

17. Po-Wei Wang, Priya L. Donti, Bryan Wilder, Zico Kolter, (2019): SATNet: Bridging Deep Learning and Logical Reasoning Using a Differentiable Satisfiability Solver, Proceedings of the 36th International Conference on Machine Learning, Long Beach, California, PMLR 97. http://proceedings.mlr.press/v97/wang19e/wang19e.pdf

18. Ries, E. (2011): The Lean Startup: How Today's Entrepreneurs Use Continuous Innovation to Create Radically Successful Businesses; Crown Business: New York, NY, USA.

19. Sara Raed Majeed, Basim Hasan Al-Majidi (2019): Analogical reasoning in architecture, Al-Qadisiyah Journal for Engineering Sciences. https://www.researchgate.net/publication/333774808_Analogical_reasoning_in_architecture

20. Syed Junaid Nawaz, Shree K. Sharma, Shurjeel Wyne, Mohammad N. Patwary, and Md Asaduzzaman, (2019): Quantum Machine Learning for 6G Communication Networks: State-of-the-Art and Vision for the Future, IEEE ACCESS, Retrieved on 03 2020, p. 4631746350, from

https://www.researchgate.net/publication/332219159_Quantum_Machine_Learning_for_6G_Communication_Networks_State-of-theArt_and_Vision_for_the_Future

21. Helsinki Rapport (2017): Finland's Age of Artificial Intelligence.Turning Finland into a leading country in the application of Artificial Intelligence. Objective and recommendations for measures, Publications of the Ministry of Economic Affairs and Employment Ministry. https://julkaisut.valtioneuvosto.fi/bitstream/handle/10024/160391/TEMrap_47_2017_verkkojulkaisu.pdf

22. (2017): TCS Global Trend Study, Part 1, Getting Smarter by the Day: How AI is Elevating the Performance of Global Companies. https://sites.tcs.com/artificial-intelligence/wp-content/uploads/TCS-GTS- how-AI- elevating-performance-global-companies.pdf

23. 23.CISCO (2018): Transforming Businesses with Artificial Intelligence. https://www.cisco.com/c/dam/en/us/solutions /collateral/ digital-transformation/ai-whitepaper.pdf

24. (2018): Artificial Intelligence. Shaping a Future New Zealand. An Analysis of the Potential Impact and Opportunity of Artificial Intelligence on New Zealand's Society and Economy. https://www.mbie.govt.nz/dmsdocument/5754-artificial-intelligence-shaping-afuture-new-zealand-pdf

25. Cigref Report (2018): Artificial Intelligence In Companies Strategies, Governance and Challenges of Data Intelligence. https://www.cigref.fr/wp/wp-content/uploads/2018/11/Cigref-Artificial-intelligence-in-companies-Strategies-governance challenges -of-data-intelligence-2018-October-EN.pdf

26. Cognizant (2018): Digital Business,Making Artificial Intelligence Responsible and Effective. https://www.cognizant.com/ whitepapers /making-ai-responsible-and-effective-codex3974.pdf

27. (2018): First special issue on The impact of Artificial Intelligence on communication networks and services, ITU Journal ICT Discoveries, Volume 1, No. 1, 03, https://www.itu.int/dms_pub/itu-s/opb/journal/S-JOURNAL-ICTF.VOL1-2018-1-PDF-E.pdf

28. (2019): 2016- 2019 Progress Report, Advancing Artificial Intelligence R\&D A Report By The Artificial Intelligence Research \& Development Inter Agency Working Group, Subcommittee on Networking \& Information Technology Research \& Development, Subcommittee on Machine Learning \& Artificial Intelligence and The Select Committee on Artificial Intelligence of the National Science \& Technology Council, USA. https://www.whitehouse.gov/wp-content/uploads/2019/11/AI-Research-and-Development-ProgressReport-2016-2019.pdf

29. (2019): Künstliche IntelligenzTechnologie, Anwendung, Gesellschaft, ISBN 978-3-662-58041-7, ISBN 978-3-662-58042-4 (eBook), DOI 10.1007/978-3-662-58042-4. https://link.springer.com/content/pdf/10.1007\%2F978-3-662-58042-4.pdf. Künstliche Intelligenz

30. (2020): Dell EMC's 2020 Server Trends \& Observations. https://www.dellemc.com/resources/en-us/asset/articles/ products /servers/2020-server-trends-and-observations-brief.pdf

31. Patricia A. Damkroger (2018): The Intersection of AI, HPC and HPDA: How Next-Generation Workflows Will Drive Tomorrow's Breakthroughs, https://www.top500.org/features/content/the-intersection-of-ai-hpc-and-hpda-how-next-generation-workflows-willdrive-tomorrows-breakthroughs/

32. Gangman Yi, Vincenzo Loia (2019): High-performance computing systems and applications for AI, Springer Science +Business Media, LLC, part of Springer Nature 2019, https://link.springer.com/article/10.1007/s11227-019-02937-z

33. (2019): 5G PPPArchitectureWorking GroupView on5G Architecture

34. https://5g-ppp.eu/wp-content/uploads/2019/07/5G-PPP-5G-Architecture-White-Paper_v3.0_PublicConsultation.pdf

35. Mengdi Wang, Chen Meng, Guoping Long1, Chuan Wu, Jun Yang1, Wei Lin, Yangqing Jia, (2019): Characterizing Deep Learning Training Workloadson Alibaba-PAI, https://arxiv.org/pdf/1910.05930.pdf

36. (2018): Intel, EnterpriseData Center, Alibaba Cloud POLARDB: An Innovative Cloud-Hosted Database,

37. https://www.intel.com.br/content/dam/www/public/us/en/documents/solution-briefs/alibaba-polardb-solution-brief.pdf 